\title{
COSYMPLECTIC HYPERSURFACES IN KAEHLERIAN MANIFOLD OF CONSTANT HOLOMORPHIC SECTIONAL CURVATURE
}

\author{
By Masafumi Okumura
}

\section{Introduction.}

An odd dimensional differentiable manifold $M^{2 n-1}$ is said to have an almost cosymplectic structure or called an almost cosymplectic manifold if there exist in $M^{2 n-1}$ a 1 -form $\omega$ and a 2 -form $\pi$ such that

$$
\omega \wedge \pi^{n-1} \neq 0,
$$

at each point of $M^{2 n-1}$ and the pair $(\omega, \pi)$ is called an almost cosymplectic structure of $M^{2 n-1}$. If, in an almost cosymplectic manifold, the 1-form $\omega$ and the 2 -form $\pi$ are both closed, that is, they satisfy the conditions

$$
d \omega=0, \quad d \pi=0,
$$

the manifold is called a cosymplectic manifold [2]. ${ }^{1)}$ The study of such manifolds has been developed by Libermann [2,3], Reeb [6] and Takizawa [10].

On the other hand, using a differential geometric method found by Sasaki [7] recently, Kurita [1], Tashiro [8], Tashiro and Tachibana [9] and the present author $[4,5]$ studied hypersurfaces of an almost complex manifold.

In the previous papers $[4,5]$, the author discussed normal almost contact hypersurface of a Kaehlerian manifold of constant holomorphic sectional curvature. In this paper, the author discuss cosymplectic hypersurfaces of Kaehlerian manifold of constant holomorphic sectional curvature.

In $\S 1$, we give first of all some preliminaries of an almost Hermitian manifold and of its hypersurface.

In $\S 2$, we define the induced almost cosymplectic structure of the hypersurface and show a certain condition for the structure to be cosymplectic.

In $\S 3$, we study non-existence of the cosymplectic hypersurface under certain conditions for the scaler curvature of a Kaehlerian manifold of constant holomorphic sectional curvature.

We devote $\S 4$ to the study of the principal curvatures of the hypersurface and, as an application, we prove that the scalar curvature of a cosymplectic hypersurface

Received October 28, 1964.

1) The numbers in the brackets refer to the bibliography at the end of the paper. 
of a Kaehlerian manifold of constant holomorphic sectional curvature is non-positive constant.

In $\S 5$, we discuss the distribution spanned by the orthogonal vectors to $\eta^{2}$ and the integrability of the distribution is proved. Moreover, in this section, we study the integral submanifold of the distribution in detail.

\section{Almost Hermitian manifold and its hypersurfaces.}

On an $2 n$-dimensional real differentiable manifold $\bar{M}^{2 n}$ with local coordinate systems $\left\{X^{k}\right\}$, if there exist a tensor field $F_{\lambda}{ }^{k}$ satisfying

$$
F_{\lambda}{ }^{\kappa} F_{\mu}{ }^{\lambda}=-\delta_{\mu}{ }^{\kappa},
$$

then the tensor field $F_{\lambda^{x}}$ is called an almost complex structure and the manifold $\bar{M}^{2 n}$ with such a structure is called an almost complex manifold. It is well known fact that an almost complex manifold $\bar{M}^{2 n}$ always admits a positive definite Riemannian metric tensor $G_{\lambda k}$ satisfying

$$
G_{\lambda \kappa} F_{\nu}{ }^{\lambda} F_{\mu}{ }^{k}=G_{\nu \mu},
$$

which is called the Hermitian metric. The pair $\left(F_{\lambda}{ }, G_{\lambda x}\right)$ with the above properties is called an almost Hermitian structure and manifold $\bar{M}^{2 n}$ almost Hermitian manifold. In an almost Hermitian manifold the covariant tensor field $F_{\lambda \kappa}$ given by $F_{\lambda \kappa}=G_{\mu \kappa} F_{\lambda^{\mu}}$ is skew symmetric and so we can construct a 2 -form

$$
\Theta=F_{\lambda \mu} d X^{\lambda} \wedge d X^{\mu},
$$

which is called the fundamental 2-form of the almost Hermitian manifold $\bar{M}^{2 n}$. We call an almost Kaehlerian manifold an almost Hermitian manifold with the closed fundamental 2-form. The condition of closedness of the fundamental 2-form is equivalent to the following tensor equation:

$$
\bar{\nabla}_{\mu} F_{\lambda \kappa}+\bar{\nabla}_{\lambda} F_{\kappa \mu}+\bar{\nabla}_{\kappa} F_{\mu \lambda}=0 \text {, }
$$

where $\bar{\nabla}$ denotes the covariant differentiation with respect to the Christoffel symbol formed from the Hermitian metric. In an almost Hermitian manifold, if the almost complex structure $F_{\lambda}{ }^{k}$ satisfies

$$
\bar{\nabla}_{\mu} F_{\lambda}{ }^{*}=0
$$

then the manifold is said to be Kaehlerian.

Let $\bar{M}^{2 n}$ be an almost Hermitian manifold with local coordinates $\left\{X^{\kappa}\right\}$ and $\left(F_{\lambda^{\kappa}}, G_{\lambda k}\right)$ be the almost Hermitian structure. A differentiable hypersurface $M^{2 n-1}$ of $\bar{M}^{2 n}$ may be represented parametrically by the equation $X^{\kappa}=X^{\kappa}\left(x^{i}\right)$.

Assuming that the hypersurface $M^{2 n-1}$ be orientable, we put $B_{i}{ }^{\kappa}=\partial_{i} X^{\kappa},\left(\partial_{i}=\partial\right.$ 
$\left(\partial x^{i}\right)$. Then, $2 n-1$ vectors $B_{i}{ }^{k}$ span the tangent hyperplane of $M^{2 n-1}$ at each point of $M^{2 n-1}$ and we denote by $C^{k}$ the unit normal vector to the hypersurface. The $2 n$ vectors $B_{i}{ }^{k}, C^{k}$ being linearly independent, we can construct the inverse of the matrix $\left(B_{i}{ }^{k}, C^{k}\right)$ which we denote by $\left(B^{i}{ }_{k}, C_{k}\right)$. Then we have the following identities.

$$
B_{i}{ }^{\lambda} B^{h}{ }_{\lambda}=\delta_{i}{ }^{h}, B_{i}{ }^{\lambda} C_{\lambda}=0, B^{i}{ }_{\lambda} C^{\lambda}=0, C^{\lambda} C_{\lambda}=1,
$$

$$
B_{i}{ }^{k} B^{i}{ }_{\lambda}+C^{\kappa} C_{\lambda}=\delta_{\lambda}{ }^{k} \text {. }
$$

From the Hermitian metric $G_{\lambda x}$ of $\bar{M}^{2 n}$, we can induce a Riemannian metric $g_{j i}$ of $\bar{M}^{2 n-1}$ by

$$
g_{j i}=G_{\lambda k} B_{j}^{\lambda} B_{i}{ }^{k} .
$$

We call this metric the induced Riemannian metic and, in the following, we always use the induced metric. If we denote by $\nabla$, the covariant differentiation along the hypersurface $M^{2 n-1}$, the Gauss and Weingarten equations for the hypersurface are respectively given by

$$
\nabla j B_{i}{ }^{\kappa}=H_{j i} C^{\kappa},
$$

$$
\nabla_{j} C_{\kappa}=-H_{j i} B^{i},
$$

where $H_{j i}$ is the second fundamental tensor of the hypersurface.

Let $\bar{R}_{\nu \mu \lambda \varepsilon}$ and $R_{k j i n}$ be the covariant components of the curvature tensors of $\bar{M}^{2 n}$ and $M^{2 n-1}$ respectively, then the following Gauss and Codazzi equations are well known.

$$
\begin{gathered}
R_{k j i h}=B_{k^{\nu}} B_{j^{\mu}} B_{i}{ }^{\lambda} B_{h^{k}} \bar{R}_{\nu \mu \lambda \kappa}+H_{j i} H_{k h}-H_{k i} H_{j h}, \\
\nabla_{k} H_{j i}-\nabla^{j} H_{k i}=B_{k^{\nu}} B_{j}{ }^{\mu} B_{i}{ }^{\lambda} C^{k} \bar{R}_{\nu \mu \lambda \kappa} .
\end{gathered}
$$

2. Induced almost cosymplectic structure of a hypersurface of an almost Hermitian manifold.

We put

$$
\begin{gathered}
\eta_{j}=B_{j}{ }^{\lambda} F_{\lambda}{ }^{k} C_{\kappa}, \\
\phi_{j k}=B_{j}{ }^{\lambda} F_{\lambda k} B_{k}{ }^{k} .
\end{gathered}
$$

Then $\phi_{j k}$ is a skew symmetric tensor defined on $M^{2 n-1}$ and by virtue of (1.6), (1.7) and (1.8) we get

$$
\phi_{j k} \eta^{k}=0
$$




$$
\phi_{j i} \phi_{h^{j}}=g_{i h}-\eta_{i} \eta_{h},\left(\phi_{h}{ }^{j}=g^{j r} \phi_{i r}\right) .
$$

From these facts, the forms defined by

$$
\omega=\eta_{i} d x^{2}, \quad \pi=\phi_{j i} d x^{j} \wedge d x^{2}
$$

satisfy the condition (0.1). Thus we know that the pair of the forms $(\omega, \pi)$ defines an almost cosymplectic structure of the hypersurface $M^{2 n-1}$. If the almost cosymplectic structure is a cosymplectic one, we call the hypersurface a cosymplectic hypersurface of $\bar{M}^{2 n}$.

From (2.1) and (2.2) we can easily see that

$$
\eta_{i} \eta^{2}=1 \text {. }
$$

Using the local coordinates, we have the equivalent conditions of cosymplecticness (0.2) as

$$
\nabla_{i} \eta_{i}-\nabla_{i} \eta_{j}=0
$$

$$
\nabla_{j} \phi_{i h}+\nabla_{i} \phi_{h j}+\nabla_{h} \phi_{j i}=0,
$$

in the hypersurface.

Differentiating (2.1) and (2.2) covariantly along the hypersurface, we have

$$
\begin{aligned}
\nabla_{j} \eta_{i} & =B_{i}^{\mu} B_{j}{ }^{\nu} \bar{\nabla}_{\nu} F_{\mu}{ }^{\lambda} C_{\lambda}-B_{i^{\mu}} F_{\mu}{ }^{2} B^{r}{ }_{\lambda} H_{j r}, \\
\nabla_{j} \phi_{i h} & =H_{j i} C^{\kappa} F_{\kappa \lambda} B_{h^{2}}+B_{i^{\mu}} B_{j}{ }^{\nu} \bar{\nabla}_{\nu} F_{\mu \lambda} B_{h^{2}}+B_{i^{\mu}} F_{\mu \lambda} H_{j h} C^{\lambda},
\end{aligned}
$$

by virtue of skew-symmetric property of $F_{\lambda k}$. Substituting (2.1) and (2.2) into the above equations, we get

$$
\begin{aligned}
\nabla_{j} \eta_{i} & =B_{i}{ }^{\mu} B_{j}{ }^{\nu} \bar{\nabla}_{\nu} F_{\mu \lambda} C^{\lambda}-\phi_{i}{ }^{r} H_{r \jmath}, \\
\nabla_{j} \phi_{i h} & =B_{i^{\mu}} B_{j}{ }^{\nu} \bar{\nabla}_{\nu} F_{\mu \lambda} B_{h}{ }^{2}+\eta_{i} H_{j h}-\eta_{h} H_{j i} .
\end{aligned}
$$

If the almost Hermitian manifold $\bar{M}^{2 n}$ is Kaehlerian, the above equations reduce to

$$
\begin{aligned}
\nabla_{j} \eta_{i} & =-\phi_{i}^{r} H_{r \jmath}, \\
\nabla_{j} \phi_{i h} & =\eta_{i} H_{j h}-\eta_{h} H_{j i} .
\end{aligned}
$$

From (2. 8) and (2.9), we see that in any hypersurface of Kaehlerian manifold, the condition (2.7) is always satisfied and that $\nabla r \eta^{r}=0$. Thus we have the

THEOREM 2.1. In order that a hypersurface of a Kaehlerian manifold is cosymplectic, it is necessary and sufficient that the vector $\eta^{2}$ is a harmonic vector. ${ }^{2}$

From this theorem, we have

2) As to harmonic vectors, we refer to Yano and Bochner [11]. 
Theorem 2.2. A totally geodesic hypersurface of Kaehlerian manifold is a cosymplectic hypersurface.

Now, in what follows, we always consider the cosymplectic hypersurface of Kaehlerian manifold. Then we have from (2.6) and (2.8)

$$
H_{j}^{r} \phi_{i r}=H_{r i} \phi_{j}^{r} \text {. }
$$

Transvecting this with $\eta^{2}$, we get

$$
H_{r i} \phi_{j}^{r} \eta^{\imath}=0
$$

from which

$$
H_{r i} \eta^{r}=\alpha \gamma_{i} \quad\left(\alpha=H_{r i} \eta^{r} \eta^{i}\right)
$$

This means that the scalar $\alpha$ is a characteristic root of the second fundamental tensor $H_{j}^{2}$ and that the vector $\eta^{2}$ is one of the corresponding characteristic vector to the root $\alpha$.

If we transvect (2.10) with $\phi_{k}{ }^{i}$, then it follows that

$$
-H_{j k}+\alpha \eta_{j} \eta_{k}=H_{r i} \phi_{j}^{r} \phi_{k}{ }^{2}
$$

and consequently

$$
H_{r}^{r}=g^{j i} H_{j i}=\alpha,
$$

because of (2.3), (2.4) and (2.5).

\section{Hypersurface of a Kaehlerian manifold of constant holomorphic sectional curvature.}

Let $\bar{M}^{2 n}$ be a Kaehlerian manifold and $X^{\kappa}$ be a vector in $\bar{M}^{2 n}$, then $F_{\lambda}{ }^{\kappa} X^{\lambda}$ is also a vector orthogonal to $X^{\kappa}$, and consequently the holomorphic sectional curvature with respect to the vector $X^{*}$ is given by

$$
K=-\frac{\bar{R}_{\nu \mu \lambda_{\kappa}} F_{\pi^{\mu}} F_{\sigma}{ }^{k} X^{\nu} X^{\pi} X^{\lambda} X^{\sigma}}{G_{\lambda \mu} X^{\lambda} X^{\mu} G_{\kappa \nu} X^{\kappa} X^{\nu}}
$$

If the holomorphic sectional curvature is always constant with respect to any vector at each point of the manifold, then we call the manifold that of constant holomorphic sectional curvature. It has been proved that the Kaehlerian manifold of constant holomorphic sectional curvature has the curvature tensor of the form: ${ }^{3)}$

$$
\bar{R}_{\nu \mu \lambda \kappa}=k\left(G_{\mu \lambda} G_{\nu \kappa}-G_{\nu \lambda} G_{\mu \kappa}+F_{\mu \lambda} F_{\nu \kappa}-F_{\nu \lambda} F_{\mu \kappa}-2 F_{\nu \mu} F_{\lambda \kappa}\right),
$$

3) Yano and Mogi [13]. 
where $k=K / 4$ is constant.

Substituting (3.2) into (1.11) and (1.12), we have

$$
R_{k j i h}=k\left(g_{j i} g_{k h}-g_{k i} g_{j h}+\phi_{k h} \phi_{j i}-\phi_{j h} \phi_{k i}-2 \phi_{k j} \phi_{i h}\right)+H_{k h} H_{j i}-H_{j h} H_{k \imath}
$$

and

$$
\nabla_{k} H_{j i}-\nabla_{j} H_{k i}=k\left(\eta_{k} \phi_{j i}-\eta_{j} \phi_{k i}-2 \phi_{k j} \eta_{i}\right),
$$

because of (2.1), (2.2) and (1.8). Consequently we have

$$
\left(\nabla_{k} H_{j i}-\nabla_{j} H_{k i}\right) \eta^{\imath}=-2 k \phi_{k j} .
$$

The Ricci tensor of the hypersurface is given by

$$
R_{j i}=k\left[(2 n+1) g_{j i}-3 \eta_{j} \eta_{i}\right]+\alpha H_{j i}-H_{r j} H_{i}{ }^{r} .
$$

Now we prove the

THEOREM 3. 1. There is no cosymplectic hypersurface in a Kaehlerian manifold of positive constant holomorphic sectional curvature.

Proof. Applying the Ricci's identity to $\eta_{i}$, we obtain

$$
\nabla j \nabla i \gamma_{h}-\nabla i \nabla_{j} \eta_{h}=-\eta_{r} R_{j i h}{ }^{r}
$$

from which

$$
\nabla^{r} \nabla r \eta_{j}=\nabla^{r} \nabla_{j} \eta_{r}=\eta^{r} R_{j}
$$

because of (2.6). Substituting (3.6) into (3.7) and making use of (2.11), we get

$$
\nabla^{r} \nabla r \eta_{j}=2(n-1) k \eta_{j}
$$

Transvecting (3.8) with $\eta^{\jmath}$ and making use of (2.5), we have at last

$$
2(n-1) k=-\nabla^{r} \eta^{\jmath} \nabla r \eta_{j}
$$

The Riemannian metic being positive definite, we have, from (3.9), $k=K / 4 \leqq 0$. This completes the proof.

If the Kaehlerian manifold is a locally Euclidean manifold, by virtue of (3.9), it follows that $\nabla i \eta_{i}=0$ and consequently $\phi_{j}{ }^{2} H_{2 r}=0$. Hence we have

$$
H_{i j}=\alpha \eta_{i} \eta_{j}
$$

Substituting (3.10) into (1. 11), we get $R_{k j i h}=0$. Thus we have proved the

Theorem 3.2. Let $E^{2 n}$ be a locally Euclidean manifold. Then, a cosymplectic hypersurface of $E^{2 n}$ is locally isometric with a Euclidean space $E^{2 n-1}$. 


\section{Principal curvatures and the scalar curvature of the hypersurface.}

In this section, assuming that $M^{2 n-1}$ be a cosymplectic hypersurface of a nonflat Kaehlerian manifold of constant holomorphic sectional curvature, we discuss the scalar curvatures and the characteristic roots of the second fundamental tensor $H_{j}{ }^{2}$. Differentiating (2.11) covariantly and making use of (2.8), we have

$$
\begin{aligned}
& \nabla_{k} H_{j i} \eta^{2}-H_{j}{ }^{2} \phi_{i}^{r} H_{r k}=\nabla k \alpha \eta_{j}+\alpha \nabla_{k} \eta_{j}, \\
& \nabla_{j} H_{k i} \eta^{2}-H_{k}{ }^{2} \phi_{i}{ }^{r} H_{r j}=\nabla_{j} \alpha \eta_{k}+\alpha \nabla_{j} \eta_{k},
\end{aligned}
$$

from which, together with (2.6), we get

$$
\left(\nabla_{k} H_{j i}-\nabla_{j} H_{k i}\right) \eta^{2}-H_{j}{ }^{2} \phi_{i}^{r} H_{r k}+H_{k}{ }^{2} \phi_{i}{ }^{r} H_{r j}=\nabla_{k} \alpha \eta_{j}-\nabla_{j} \alpha \eta_{k} .
$$

If we substitute (3.5) into (4.1), it follows that

$$
-2 k \phi_{k j}+2 H_{k}{ }^{2} H_{\imath s} \phi_{j}^{s}=\nabla k \alpha \eta_{j}-\nabla j \alpha \eta_{k},
$$

because of (2.8) and (2.6). Transvecting (4.2) with $\eta^{3}$, we have

$$
\nabla_{j} \alpha=\beta \eta_{j}, \quad\left(\beta=\eta^{r} \nabla_{r} \alpha\right),
$$

and so, from (4.2), we get

$$
H_{k}^{\imath} H_{\imath s} \phi_{j}^{s}=k \phi_{k \jmath}
$$

from which

$$
H_{k}^{\imath} H_{\imath j}+k g_{k j}-\left(k+\alpha^{2}\right) \eta_{k} \eta_{j}=0,
$$

which implies that

$$
H^{j i} H_{j i}=\alpha^{2}-2(n-1) k
$$

Now, we prove the

THEOREM 4.1. In a cosymplectic hypersurface of a Kaehlerian manifold of constant holomorphic sectional curvature, the scalar curvature is a non-positive constant.

Proof. From (2.12) and (3.6), it follows that

$$
R=4\left(n^{2}-1\right) k+\alpha^{2}-H_{j i} H^{j i} .
$$

Substituting (4.5) into (4.6), we have

$$
R=2(n-1)(2 n+3) k,
$$

and consequently we get $R=$ const. $\leqq 0$ because of Theorem 3.1 . This completes the proof. 
Now, we treat to express the second fundamental tensor in matrix form. Let $\lambda$ be a characteristic root of the second fundamental tensor $H_{j}{ }^{2}$ which is distinct to the one which corresponds to $\eta^{2}$ and $v^{2}$ be a corresponding characteristic vector to the root $\lambda$. Then we have

$$
H_{j}^{\imath} v^{\jmath}=\lambda v^{2} .
$$

Transvecting (4.4) with $v^{k}$ and making use of orthogonality of $v^{k}$ to $\eta^{k}$, we get

$$
\left(\lambda^{2}+k\right) v^{\jmath}=0 .
$$

Since it has been shown that $\alpha$ is a characteristic root of the second fundamental tensor $H_{j}^{2}$ and that $\eta^{2}$ is a corresponding characteristic vector to $\alpha$, we have

THeOREM 4.2. Let $M^{2 n-1}$ be a cosymplectic hypersurface in a Kaehlerian manifold of constant holomorphic sectional curvature, then $M^{2 n-1}$ admits at most three distinct principal curvatures.

When the hypersurface $M^{2 n-1}$ admits only one principal curvature $\alpha$, then $M^{2 n-1}$ is a totally umbilical hypersurface and so the Kaehlerian manifold $\bar{M}^{2 n}$ is locally Euclidean. ${ }^{4)}$ Since we assume that $\bar{M}^{2 n}$ be non-Euclidean, it follows that the hypersurface admits two or three distinct principal curvatures. This fact, together with (2.12), means that with respect to a suitable orthonormal frame the second fundamental tensor $H_{j}{ }^{2}$ has the following components:

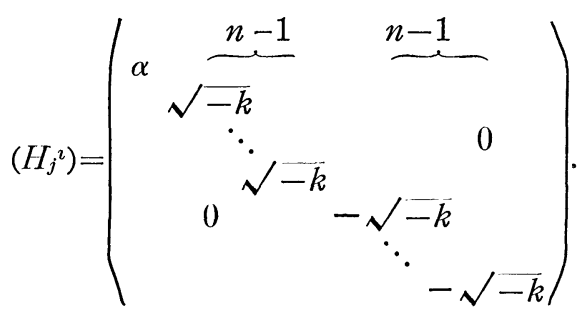

\section{A certain distribution and its integral submanifold.}

Let $M^{2 n-1}$ be a cosymplectic hypersurface of an almost Hermitian manifold and $D$ be the distribution spanned by the all vectors orthogonal to $\eta^{2}$. Then we can easily see that $D$ is an integrable distribution. From this fact, we know that through each point of the hypersurface there passes a unique integral submanifold of the distribution $D$. In this section, we speak of this integral submanifold.

Denote by $X_{a}{ }^{\imath}(a=1, \cdots, 2 n-2)$ the mutually orthonormal contravariant vectors in the distribution $D$. Then $2 n-1$ vectors $\eta^{2}, X_{a}{ }^{2}$ being linearly independent, we can construct the inverse of the matrix $\left(\eta^{\imath}, X_{a}^{i}\right)$ which we denote by $\left(\eta_{i}, X^{a}{ }_{\imath}\right)$. It

4) Tashiro and Tachibana [9]. 
is easily see that the $\eta_{i}$ in the inverse matrix is identical with that used in the previous discussions. From this we have the identities:

$$
\begin{aligned}
& X_{b}{ }^{\imath} X^{a}{ }_{i}=\delta_{b}{ }^{a}, \quad X_{b}{ }^{\imath} \eta_{i}=0, \quad \eta^{i} X^{a}{ }^{a}=0, \quad \eta_{i} \eta^{i}=1, \\
& X_{a}{ }^{\imath} X^{a}{ }_{j}+\eta^{\imath} \eta_{j}=\delta_{j}{ }^{\imath} \text {. }
\end{aligned}
$$

If we put

$$
{ }^{\prime} f_{b}{ }^{a}=\phi_{j}{ }^{i} X_{b}{ }^{j} X^{a}{ }_{\imath},
$$

${ }^{\prime} f_{b} a$ is a tensor defined in the integral submanifold of the distribution. Making use of (5.1) and (5. 2), we can obviously see that ' $f_{b}{ }^{a}$ defines an almost complex structure of the integral submanifold and that the metric defined by

$$
{ }^{\prime} g_{b a}=g_{j i} X_{b}^{j} X_{a}^{\imath}
$$

is the Hermitian metric.

We put

$$
{ }^{\prime} \Gamma_{b}{ }^{a}{ }_{c}=\left(X_{b}{ }^{j} X_{c}{ }^{k}\left\{{ }_{\jmath}{ }^{i} k\right\}+X_{b}{ }^{j} \partial_{\jmath} X_{c}{ }^{i}\right) X^{a}{ }_{\imath},
$$

then the covariant derivative of ' $f_{b a}={ }^{\prime} g_{a c} f_{b}{ }^{c}$ along the integral submanifold of $D$ becomes

$$
{ }^{\prime} \nabla_{c}^{\prime} f_{b a}=\nabla_{k} \phi_{j i} X_{c}{ }^{k} X_{b}^{j} X_{a}{ }^{2}+\phi_{j i}{ }^{\prime} H_{c b} \eta^{j} X_{a}{ }^{2}+\phi_{j i} X_{b}{ }^{\prime} H_{a c} \eta^{\imath}{ }^{5)}
$$

$$
=\nabla_{k} \phi_{j i} X_{c}^{k} X_{b}^{j} X_{a}^{\imath},
$$

where ' $H_{a b}$ denotes the second fundamental tensor of the integral submanifold of $D$. From this we get

$$
{ }^{\prime} \nabla c f_{b a}+{ }^{\prime} \nabla{ }^{\prime} f_{a c}+{ }^{\prime} \nabla a f_{c b}=0,
$$

because of (2.7). This proves the following theorem by accordance of (1.4).

THEOREM 5.1. In a cosymplectic hypersurface of an almost Hermitian manifold, the integral submanifold of the distribution $D$ which spanned by the all orthogonal vectors to $\eta^{i}$ is an almost Kaehlerian manifold.

If the Hermitian manifold of Theorem 5.1 is Kaehlerian, we substitute (2.9) into (5.6) and obtain

$$
{ }^{\prime} \nabla c f_{b a}=0,
$$

which implies the

THEOREM 5.2. In a cosymplectic hypersurface of a Kaehlerian manifold, the integral submanifold of $D$ is also a Kaehlerian manifold.

5) Yano and Davies [12]. 
Now, assuming that $M^{2 n-1}$ be a cosymplectic hypersurface of Kaehlerian manifold of constant holomorphic sectional curvature, we consider the integral submanifold of the distribution $D$.

LEMMA 5. 3. Let $v^{2}$ be an orthogonal vector to $\eta^{2}$. If $v^{2}$ is a characteristic vector corresponding to the root $\varepsilon \sqrt{-k}(\varepsilon= \pm 1)$ of the second fundamental tensor of $M^{2 n-1}$, then the vector $\phi_{j}{ }^{2} v^{j}$ is a characteristic vector corresponding to the root $-\varepsilon \sqrt{-k}$.

Proof. According to (2.10), we have

$$
H_{j}^{r} \phi_{i r} v^{\jmath}=H H_{r i} \phi_{j}^{r} v^{\jmath},
$$

from which

$$
-\varepsilon \sqrt{-k} \phi_{r}^{\imath} v^{r}=H_{r}^{\imath} \phi_{j}^{r} v^{\jmath}
$$

This proves the lemma.

Denote by $D_{+}$and $D_{-}$be the distributions spanned by the orthogonal vectors to $\eta^{2}$ which corresponding to the characteristic roots $\sqrt{-k}$ and $-\sqrt{-k}$ of the second fundamental tensor $H_{j}{ }^{2}$ respectively. Then it follows that

$$
D(p)=D_{+}(p) \oplus D_{-}(p) \quad(\text { derect sum }),
$$

at each point of the integral submanifold of $D$. As to the distributions $D_{+}$and $D_{-}$, we show the

Theorem 5.4. The distributions $D_{+}$and $D_{-}$are both integrable.

Proof. Since another case can be proved quite analogously, we only prove that $D_{+}$is integrable. Let $u^{\imath}$ and $v^{\imath}$ be two arbitrary vectors belonging to $D_{+}$. Then we have

$$
H_{j}^{\imath} u^{\jmath}=\sqrt{-k} u^{\imath}, \quad H_{j}^{\imath} v^{\jmath}=\sqrt{-k} v^{2},
$$

from which

$$
\begin{aligned}
& \nabla_{k} H_{j}^{\imath} v^{k} u^{\jmath}+H_{j} v^{k} \nabla_{k} u^{\jmath}=\sqrt{-k} v^{k} \nabla_{k} u^{2}, \\
& \nabla_{k} H_{j}^{\imath} u^{k} v^{\jmath}+H_{j}^{\imath} u^{k} \nabla_{k} v^{\jmath}=\sqrt{-k} u^{k} \nabla_{k} v^{2},
\end{aligned}
$$

and consequently

$$
\left(\nabla_{k} H_{j}{ }^{2}-\nabla_{j} H_{k}{ }^{i}\right) u^{k} v^{\jmath}+H_{j}{ }^{i}[u, v]^{\jmath}=\sqrt{ } \overline{-k}[u, v]^{i} .
$$

Substituting (3.4) into (5.8), we get

$$
k\left(\eta_{k} \phi_{j}{ }^{2}-\eta_{j} \phi_{k}{ }^{2}-2 \phi_{k j} \eta^{i}\right) u^{k} v^{\jmath}+H_{j}{ }^{i}[u, v]^{\jmath}=\sqrt{-k}[u, v]^{i} .
$$


However, by virtue of Lemma 5.3, the first term of the above equation vanishes. This means that the bracket of the vectors $u^{2}$ and $v^{2}$ belongs to $D_{+}$. This completes the proof.

From Theorem 5. 4 we know that through each point of $M^{2 n-1}$ there pass the integral submanifolds of $D_{+}$and $D_{-}$. Since the distributions $D_{+}$and $D_{-}$are complement in $D$, the product of these integral submanifolds is integral submanifold of $D$. Thus we have the following

THEOREM 5.5. In a cosymplectic hypersurface of Kaehlerian manifold of constant holomorphic sectional curvature, the integral submanifold of $D$ is a locally product manifold.

\section{Bibliography}

[1] Kurita, M., On normal contact metric manifolds. Journ. Math. Soc. Japan 15 (1963), 304-318.

[2] Libermann, P., Sur les automorphismes infinitésimaux des structures symplectiques et des structures de contact. Coll. de géom. diff. globale. CBRM (1959), $37-59$.

[ 3 ] Libermann, P., Sur quelques exemples de structures pfaffiennes et presque cosymplectiques. Ann. di Mat. 60 (1962), 153-172.

[4] Okumura, M., Certain almost contact hypersurfaces in Euclidean spaces. Kōdai Math. Sem. Rep. 16 (1964), 44-54.

[5] OKumura, M., Certain almost contact hypersurfaces in Kaehlerian manifolds of constant holomorphic sectional curvatures. Tôhoku Math. Journ. 16 (1964), 270-284.

[6] ReEB, G., Sur les solutions périodiques des systèmes différentiels perturbés. Canad. Journ. Math. (1951), 339-362.

[7] SASAKI, S., On differentiable manifolds with certain structures which are closely related to almost contact structure I. Tôhoku Math. Journ. 12 (1960), 459-476.

[8] TASHIRo, Y., On contact structure of hypersurfaces in complex manifolds I. Tôhoku Math. Journ. 15 (1963), 62-78.

[9] Tashiro, Y., and S. Tachibana, On Fubinian and C-Fubinian manifolds. Kōdai Math. Sem. Rep. 15 (1963), 176-183.

[10] Takizawa, S., On contact structures of real and complex manifolds. Tôhoku Math. Journ. 15 (1963), 227-252.

[11] Yano, K., and S. Bochner, Curvature and Betti numbers. Ann. of Math. Studies 32 (1953).

[12] Yano, K., And E. T. Davies, Contact tensor calculus. Ann. di Mat. 37 (1954), $1-36$.

[13] Yano, K., AND I. Mogi, On real representations of Kaehlerian manifolds. Ann. of Math. 61 (1955), 170-188.

Department of Mathematics, Tokyo Institute of Technology. 